\title{
Selective depletion of mouse kidney proximal straight tubule cells causes acute kidney injury
}

\author{
Michiko Sekine $\cdot$ Toshiaki Monkawa Ryuji Morizane $\cdot$ Kunie Matsuoka \\ Choji Taya • Yoshiko Akita $\cdot$ Kensuke Joh · Hiroshi Itoh • Matsuhiko Hayashi • \\ Yoshiaki Kikkawa $\cdot$ Kenji Kohno $\cdot$ Akemi Suzuki $\cdot$ Hiromichi Yonekawa
}

Received: 19 May 2010/Accepted: 8 March 2011/Published online: 24 March 2011

(C) The Author(s) 2011. This article is published with open access at Springerlink.com

\begin{abstract}
The proximal straight tubule (S3 segment) of the kidney is highly susceptible to ischemia and toxic insults but has a remarkable capacity to repair its structure and function. In response to such injuries, complex processes take place to regenerate the epithelial cells of the S3 segment; however, the precise molecular mechanisms of this regeneration are still being investigated. By applying the "toxin receptor mediated cell knockout" method under the control of the S3 segmentspecific promoter/enhancer, Gsl5, which drives core $2 \beta$ $1,6-N$-acetylglucosaminyltransferase gene expression, we established a transgenic mouse line expressing the human diphtheria toxin (DT) receptor only in the S3 segment. The administration of DT to these transgenic
\end{abstract}

M. Sekine - K. Matsuoka · C. Taya - Y. Akita .

H. Yonekawa $(\square)$

Department of Laboratory Animal Science,

Tokyo Metropolitan Institute of Medical Science,

2-1-6 Kami-kitazawa, Setagaya-ku,

Tokyo 156-8506, Japan

e-mail: yonekawa-hr@igakuken.or.jp

T. Monkawa $\cdot$ R. Morizane $\cdot$ H. Itoh

Department of Internal Medicine, Keio University School

of Medicine, Tokyo 160-8582, Japan

K. Joh

Clinical Research Center, Chiba-East National Hospital, Chiba 260-8712, Japan

M. Hayashi

Apheresis and Dialysis Center, Keio University School of Medicine, Tokyo 160-8582, Japan mice caused the selective ablation of $\mathrm{S} 3$ segment cells in a dose-dependent manner, and transgenic mice exhibited polyuria containing serum albumin and subsequently developed oliguria. An increase in the concentration of blood urea nitrogen was also observed, and the peak BUN levels occurred 3-7 days after DT administration. Histological analysis revealed that the most severe injury occurred in the S3 segments of the proximal tubule, in which tubular cells were exfoliated into the tubular lumen. In addition, aquaporin 7 , which is localized exclusively to the S3 segment, was diminished. These results indicate that this transgenic mouse can suffer acute kidney injury (AKI) caused by S3 segment-specific damage after DT administration. This transgenic line

\footnotetext{
Y. Kikkawa

Department of Bioproduction, Faculty of Bioindustry, Tokyo University of Agriculture, Hokkaido 099-2493, Japan

K. Kohno

Graduate School of Biological Sciences, Nara Institute of Science and Technology, Nara 630-0101, Japan

A. Suzuki Institute of Glycoscience, Tokai University, Kanagawa 259-1292, Japan
} 
offers an excellent model to uncover the mechanisms of $\mathrm{AKI}$ and its rapid recovery.

Keywords Kidney proximal straight tubules . Transgenic mouse - Diphtheria toxin receptor . Acute kidney injury (acute renal failure)

\section{Introduction}

Kidney is an important organ to maintain body fluid homeostasis and various cell types, such as glomeruli, tubules, and collecting ducts, function in a highly coordinated manner. In such renal cells, proximal tubules play important roles on the reabsorption of salts, glucose, amino acids and water from glomerular filterate. They consist of an initial convoluted portion (S1 and S2 segments) followed by a straight portion (S3 segment). The epithelial cells of the tubules have an apical membrane with densely packed microvilli that increase the area of their luminal surface and facilitate the rate of reabsorption. Studies in experimental animal models of renal ischemia-reperfusion have established that the S3 segment of the proximal tubule is the most susceptible to injury (Witzgall et al. 1994; Cristofori et al. 2007). The S3 segment also has a remarkable capacity for regeneration. Slow-cycling cells, which are characteristic of stem cells, were identified in the S3 segment and may participate in regeneration (Maeshima et al. 2003). Hence, the S3 segment is considered a specific region responsible for the regeneration of the kidney; however, the precise mechanisms of this process are not known.

To study the in vivo functions of the S3 segment, we generated transgenic mice using the toxin receptor-mediated cell knockout (TRECK) method. The TRECK method (Saito et al. 2001) was developed to eliminate particular types of cells from a target organ. The principle of this method is to utilize the difference in susceptibility to diphtheria toxin (DT) between human and mouse cells. The diphtheria toxin receptor (DTR) is encoded by human heparin-binding epidermal growth factor-like protein (hHB-EGF, subsequently referred to as hDTR). Its mouse homo$\log$, mHB-EGF, has very low affinity for DT because the amino acids critical for DT binding are missing (Abraham et al. 1993; Hooper and Eidels 1996;
Mitamura et al. 1995). Thus, mouse cells are much more resistant to DT than human cells. A construct containing the hDTR gene with a cell-specific promoter/enhancer can be introduced into a suitable inbred mouse to produce transgenic (TRECK-Tg) mice. When DT is administered to TRECK-Tg mice, DT binds to its receptor expressed on the surface of the target cells and is incorporated into the cells by pinocytosis. Consequently, the incorporated DT inactivates polypeptide elongation factor 2 (EF-2), inhibits protein synthesis and results in apoptotic cell death (Honjo et al. 1968). Thus, only cells that express hDTR in TRECK-Tg mice can be depleted by DT administration in vivo.

To specifically eliminate S3 segment cells in the transgenic mouse kidney, we used the S3 segment cell-specific promoter/enhancer Gsl5. Gsl5 regulates the expression of glycolipids and glycoproteins that contain an $\mathrm{Le}^{\mathrm{X}}$ epitope in mouse kidney through transcriptional regulation of the core $2 \beta-1,6-N$ acetylglucosaminyltransferase (c2GnT) gene in a tissue-specific manner. While the c2GnT gene has 6 exons and 3 alternatively spliced transcripts, Gsl5 is responsible only for the transcription of the kidneyspecific mRNA, which is expressed in the S3 segment. We previously found that $G s l 5$ is a cis element located $5.5 \mathrm{~kb}$ upstream of the $\mathrm{c} 2 \mathrm{GnT}$ gene (Sekine et al. 2006). Here, we report the establishment of a C57BL/6-Gsl5 TRECK-Tg mouse line that causes S3 segment cell-specific depletion following DT administration by introducing a minigene composed of the cis-acting element and the hDTR gene.

\section{Materials and methods}

Gsl5-hDTR construct for transgenesis

Using the BAC clone $367 \mathrm{O} 1$ (Research Genetics/ Invitrogen) including the $\mathrm{c} 2 \mathrm{GnT}$ gene as a template, a PCR product containing Gsl5, a cis-regulatory element for kidney-specific expression of the c2GnT gene (Sekine et al. 2006) was amplified with the GNT-103 (5'-AAAGCATTACTGTTCCCCGAGGT TC- $\left.3^{\prime}\right)$ and GNT-170Xho (5'-GATCTCGAGAGTT CAAATCCCAGCACCCA-3') primers and digested with XhoI. Another PCR product containing the $5^{\prime}$ end of exon 1 of the $\mathrm{c} 2 \mathrm{GnT}$ gene and the flanking region just adjacent to exon 1 was amplified with the 
GNT-22Xho ( $5^{\prime}$-ATCCTCGAGAGTCCCAGGTTTT CCGTGTT- $3^{\prime}$ ) and GNT-23Eco (5'-GCTGAATTCT TGGGTTCCAGGTTCTTTCC- $3^{\prime}$ ) primers and digested with $X h o I$ and EcoRI. These fragments were purified using the QIAquick gel extraction kit (QIAGEN). After digestion with EcoRI and SalI, the fragment including the hDTR gene was excised from the pMS7 plasmid (Saito et al. 2001) and ligated with the two PCR fragments described above. The Gsl5-hDTR construct was then PCR amplified using the GNT-103 and DTR-R1 (5'-AGAGGTAGCAGTCCCCAGCC GATTC- $3^{\prime}$ ) primers with the ligated DNA as a template and purified using the QIAquick gel extraction kit.

Generation of transgenic mice

$\mathrm{Tg}$ mice were generated by microinjection of the DNA construct into pronucleus stage oocytes from C57BL/6 mice. Genomic DNA was purified from tail biopsies using a standard procedure. To screen the candidate mice, PCR analysis of genomic DNA was performed with the DTR-F1 (5'-TCCTCCAAGCC ACAAGCACTG- $\left.3^{\prime}\right)$ and DTR-R2 (5'-AGACAGAT GACAGCACCACAG- $3^{\prime}$ ) primers.

All mouse experiments were approved by the Institutional Animal Experiment Committee. The Tg line reported, C57BL/6-Gsl5 TRECK Tg, was deposited in and available from the RIKEN BioResource Center (Ibaraki, Japan).

\section{RT-PCR}

Kidney-specific expression of hDTR mRNA was analyzed by RT-PCR. A PCR product was amplified using the DTR-F1 and DTR-R2 primers after reverse transcription of total RNA purified from various tissues. To ensure that the PCR product was not derived from contaminating genomic DNA, a negative control reaction was carried out without reverse transcriptase in the mixture.

\section{Antibodies}

In this study, we used primary antibodies for antihDTR (goat, R\&D Systems), anti-aquaporin7 (AQP7, rabbit, kindly donated from Dr. Sei Sasaki), antimegalin (rabbit, used in our previous paper, Sekine et al. 2006), anti-aquaporin2 (AQP2, rabbit, SIGMA),
anti-Tamm-Horsfall Protein (THP, sheep, AbD Serotec), and anti-podocin (rabbit, SIGMA). As secondary antibodies, biotin- or Alexa Fluor 488-conjugated anti-goat IgG and Alexa Fluor 568- or 594-conjugated anti-rabbit or anti-sheep antibodies (Invitrogen) were used for immunohistochemistry, and HRPconjugated anti-goat (Santa Cruz) or anti-rabbit (GE healthcare) antibodies for western blotting analysis.

Immunoprecipitation western blotting (IP/WB)

Using ExactaCruz ${ }^{\text {TM }}$ product (Santa Cruz Biotech.), we detected hDTR protein by western blotting analysis according to the manufacturer's instructions. Briefly, each mouse kidney was homogenized in Buffer A $(0.25 \mathrm{M}$ sucrose, $0.2 \mathrm{M} \mathrm{KCl}, 0.5 \mathrm{mM}$ dithiothreitol, $0.1 \mathrm{mM}$ PMSF, $2 \mu \mathrm{g} / \mathrm{ml}$ aprotinin, $1 \mu \mathrm{g} / \mathrm{ml}$ pepstatin A, $1 \mu \mathrm{g} / \mathrm{ml}$ leupeptin, and $1 \mathrm{mM}$ sodium orthovanadate) and then centrifuged at $650 \times g$ for $5 \mathrm{~min}$. The supernatant was transferred to another tube and centrifuged again at $11,000 \times g$ for $10 \mathrm{~min}$. The supernatant was removed, and the membrane fraction was solubilized in $1 \times$ IP buffer (50 mM Tris- $\mathrm{HCl}, \mathrm{pH} 7.4,150 \mathrm{mM} \mathrm{NaCl}, 0.5 \%$ Triton X-100, $0.5 \mathrm{mM}$ dithiothreitol, 0.1 mM PMSF, $2 \mu \mathrm{g} / \mathrm{ml}$ aprotinin, $1 \mu \mathrm{g} / \mathrm{ml}$ pepstatin $\mathrm{A}, 1 \mu \mathrm{g} / \mathrm{ml}$ leupeptin, and $1 \mathrm{mM}$ sodium orthovanadate), added to the suspended $(25 \%, \mathrm{v} / \mathrm{v})$ preclearing matrix (Preclearing Matrix D) and incubated for $1 \mathrm{~h}$ at $4{ }^{\circ} \mathrm{C}$ while rotating. To form the IP antibody-IP matrix complex, anti-hDTR antibody and the suspended $(25 \%, v / v)$ IP matrix (ExactaCruz D) were incubated for at least $1 \mathrm{~h}$ at $4^{\circ} \mathrm{C}$.

Precleared lysate was transferred into the washed IP antibody-IP matrix complex and rotated overnight at $4^{\circ} \mathrm{C}$. After incubation, the precipitated complex was washed with $1 \mathrm{X}$ IP buffer 4 times, resuspended in $3 \mathrm{X}$ electrophoresis buffer, and boiled for $5 \mathrm{~min}$. After a brief spin, the supernatant was loaded onto an SDS-polyacrylamide gel and electrophoresed. We performed western blotting with the same primary antibody (goat anti-hDTR) as used for the immunoprecipitation. To confirm the specificity of the band, the pre-absorbed antibody with the recombinant hDTR (carrier-free, R\&D Systems) was also applied. The secondary antibody was an HRP-conjugated antigoat IgG antibody, and the ECL Advance system (GE healthcare) was employed for visualization. 
Administration of diphtheria toxin (DT)

DT was purified as reported previously (Saito et al. 2001). DT was dissolved in PBS and injected intraperitoneally into male $\mathrm{Tg}$ mice at 8 weeks old, at various doses such as $1(n=5), 10(n=4), 50(n=5), 75$ $(n=6)$ and $100(n=5) \mu \mathrm{g} / \mathrm{kg}$ body weight. As a negative control, $100(n=5) \mu \mathrm{g} / \mathrm{kg}$ was administered to WT littermates. Blood and urine were collected periodically from these $\mathrm{Tg}$ and WT mice. A portion of sediments excreted into the urine of Tg mice after DT administration was collected on a glass slide, fixed with methanol, and stained with Giemsa dye solution. Another portion was fixed with $4 \%$ paraformaldehyde in PBS, immersed in Starting Block ${ }^{\mathrm{TM}}$ Blocking buffer (Pierce), and then incubated with rabbit anti-AQP7 antibody, which was generously supplied by Dr. Sei Sasaki (Tokyo Medical and Dental University), and AlexaFluor594-conjugated anti-rabbit IgG antibody. The concentrations of BUN and urinary creatinine were determined with the Urea NB kit and Creatinine TestWako (both from Wako Pure Chem.), respectively. Urinary protein was determined by the Bradford assay (Bio-Rad). Aliquots of urine samples were subjected to SDS-PAGE electrophoresis and stained with Coomassie brilliant blue (CBB), and excreted protein bands were excised from the gel. After the gel pieces were washed and dried, in-gel digestion was performed with trypsin in $50 \mathrm{mM}$ ammonium bicarbonate at $37^{\circ} \mathrm{C}$ overnight. Aliquots of the digested peptides were analyzed by matrix-assisted laser desorption ionization tandem time-of-flight mass spectrometry (4800 MALDI TOF/TOF, Applied Biosystems Ltd.) (Akita et al. 2007).

Histology and immunohistochemistry

The Tg and WT mice were anesthetized with diethyl ether and sacrificed by cervical dislocation. Kidneys were then removed, fixed in $4 \%$ paraformaldehyde in PBS (pH 7.4), embedded in paraffin and sliced into $4 \mu \mathrm{m}$ serial sections.

To identify hDTR, the sections were incubated with goat anti-hDTR antibody as a primary antibody and biotin-conjugated anti-goat IgG as a secondary antibody. The Vectastain ABC (Vector) and Metalenhanced DAB Substrate (Pierce) kits were used for visualization.

After the administration of DT to the Tg mice at doses of 50,75 , and $100 \mu \mathrm{g} / \mathrm{kg}$ body weight and to
WT littermates at a dose of $100 \mu \mathrm{g} / \mathrm{kg}$ body weight, the mice $(n=4-6)$ were sacrificed on days $1,3,4,6$, 14, and 28. Paraffin sections of the kidney were stained with hematoxylin and eosin (HE) and periodic acid-Schiff (PAS) for histological examination.

For immunohistochemistry, the kidney were fixed

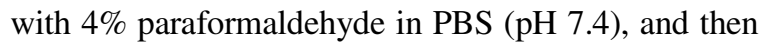
serially sliced into sections of $100 \mu \mathrm{m}$ thick using a vivratome in ice-cold PBS. After blocking in 2\% Blocking reagent(Roche Applied Science)in PBS for $1 \mathrm{~h}$ at room temperature (RT), free-floating sections were incubated overnight at $4{ }^{\circ} \mathrm{C}$ with primary antibody solutions diluted in PBS containing 0.1\% Triton X-100. We used antibodies against the hDTR (1:20) and kidney-specific markers (AQP7, 1:100; megalin, 1:50; AQP2, 1:50; THP, 1:25; podocin, 1:40) for doublelabeling experiments. After washing in PBS, the sections were incubated for $1 \mathrm{~h}$ at RT with secondary antibodies. The sections were then thoroughly rinsed in PBS, mounted in PermaFlur aqueous mounting medium (Thermo Scientific) and observed under a TCS SP5 confocal laser-scanning microscope (Leica).

Western blotting analysis of hDTR and megalin

Three days after DT administration to $\mathrm{Tg}$ mice at a dose of $75 \mu \mathrm{g} / \mathrm{kg}$, the membrane fraction of the kidney was isolated according to a method described before. For the detection of megalin, five $\mu \mathrm{g}$ of proteins were analyzed using anti-megalin antibody. For hDTR, the amount equivalent to one-eighth of the kidney was purified with immunoprecipitation and analyzed. A Tg mouse without DT administration was used as the control.

For quantitative western blot analyses, the chemiluminescently labeled bands were quantified with a LAS-3000 luminescent image analyzer (FUJIFILM) and MultiGauge Ver.3.0 software (FUJIFILM), and the expression level of both proteins of the control mouse was assigned as an arbitrary value of 10 .

\section{Results}

Generation and characterization of C57BL/6-Gsl5 TRECK transgenic mice

Figure 1A shows the Gsl5-hDTR construct, which consists of three parts: the kidney proximal tubule 


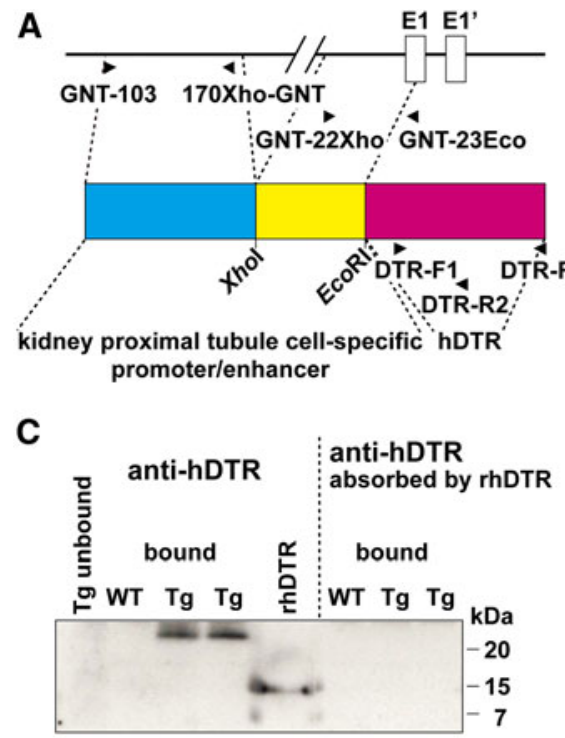

E
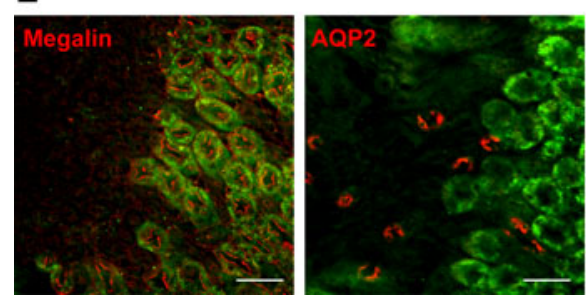

Fig. 1 Generation and analyses of the B6-Gsl5 TRECK transgenic (Tg) mice. A The Gsl5-hDTR construct contains kidney proximal tubule cell-specific promoter/enhancer regions amplified by PCR with the BAC367O1 clone as a template and the coding sequence of hDTR obtained from the pMS7 plasmid (Saito et al. 2001). E1 and E1' are exons of the c2GnT gene in the BAC. Arrowheads show the PCR primers. B RT-PCR analysis of various organs in a $\mathrm{Tg}$ mouse. To detect the expression of hDTR mRNA, DTR-F1 and DTR-R2 primers were used. RT, reverse transcription; Sub. Gl., submaxillary gland. C Immunoprecipitation-western blotting. Mouse kidney membrane fractions were immunoprecipitated with anti-hDTR

cell-specific promoter/enhancer (Gsl5, a cis element), the 5' upstream region adjacent to exon 1 of the c2GnT gene, and the hDTR gene. The sequence of the construct and, in particular, the flanking regions between the kidney S3 segment-specific promoter/ enhancer and the hDTR coding sequence were confirmed by DNA sequencing. By introducing the construct into the C57BL/6 background, two lines of C57BL/6-Gsl5 TRECK transgenic (B6-Gsl5 TRECK-Tg) mice were generated. RT-PCR analysis indicated that one of the established lines expressed a highly detectable amount of hDTR mRNA in the

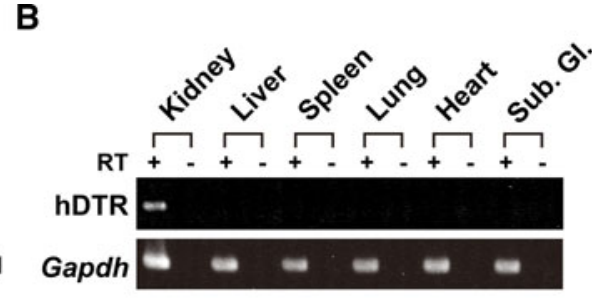

D
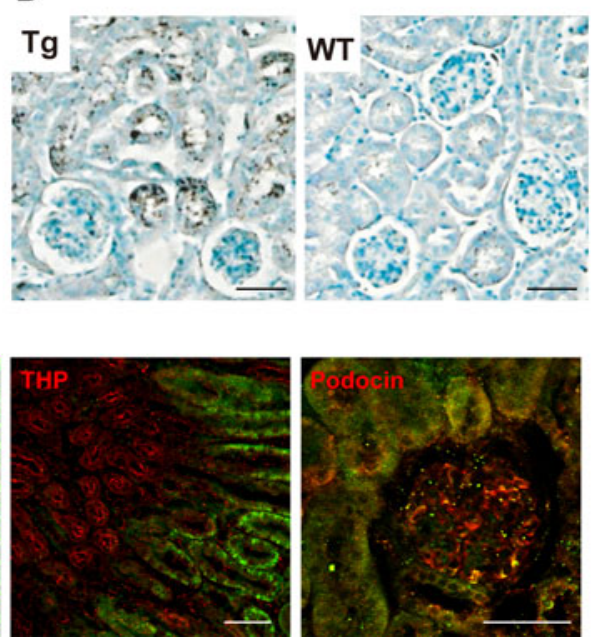

antibody. Western blotting analysis with anti-hDTR antibody showed a specific band in the fractions of the $\mathrm{Tg}$ mice bound to the IP complex, but not for the wild-type (WT) mouse or for the unbound fraction of the $\mathrm{Tg}$ mouse. rhDTR, recombinant human DTR. D Immunohistochemical study of the kidneys of $\mathrm{Tg}$ and WT mice. Human DTR protein was stained dark brown with metal-enhanced DAB reagent and the sections were counterstained with toluidine blue. The bar indicates $50 \mu \mathrm{m}$. E Immunohistochemical staining of a Tg mouse kidney. The sections were double-labeled with hDTR (green) and kidney segment specific antibodies (red; megalin, AQP2, THP and podocin). Scale bars $=50 \mu \mathrm{m}$

kidney, while the other line expressed very little transgenic mRNA. Therefore, we used the former line in this study. The Tg mice did not show any apparent morphological and functional abnormalities without the administration of DT. The hDTR mRNA expression was completely tissue-specific in $\mathrm{Tg}$ mice, and the PCR product derived from hDTR was detected only in the kidney and not in the liver, spleen, lung, heart, or submaxillary gland (Fig. 1B).

Immunoprecipitation-western blotting (IP/WB) with the anti-hDTR antibody showed a specific band in the Tg mouse kidney (Fig. 1C). Neither the non- 
precipitated fraction of the Tg mouse kidney nor the precipitated fraction of wild type (WT) mouse kidney showed such a band of approximately $20 \mathrm{kDa}$. The recombinant hDTR used as a standard migrated as an approximately $12 \mathrm{kDa}$ band on SDS-PAGE. The band in the Tg mouse kidney, however, showed lower mobility than the recombinant hDTR. The natural protein has an apparent molecular mass of $19-23 \mathrm{kDa}$ as a result of post-translational modification (Higashiyama et al. 1992), which is consistent with our data. The specificity of the band was confirmed by using a pre-absorbed anti-hDTR antibody that did not react with hDTR. The amount loaded into each lane was equivalent to one fourth of a kidney, suggesting that the transgene was weakly expressed.

Immunohistochemical observation revealed that hDTR was localized to the S3 segment of the proximal tubules but was not present in other regions, including the glomeruli, in the Tg kidney (Fig. 1D). Double-labeling with hDTR and marker proteins for other regions such as AQP2 for collecting ducts, THP for thick ascending limb (TAL) and podocin for glomurulus, also showed that hDTR did not colocalized with these proteins, whereas megalin, expressed in proximal tubule cells was localized with hDTR (Fig. 1E).

These results suggest that Gsl5, the responsible element for the S3 segment-specific transcription of the c2GnT gene, functioned correctly to express hDTR in a tissue-specific manner. Furthermore, the B6-Gsl5 TRECK-Tg mice can now be used for in vivo depletion of $\mathrm{S} 3$ segment cells in TRECK experiments.

\section{S3 segment-specific injury in TRECK experiments}

When administered at doses of more than $50 \mu \mathrm{g} / \mathrm{kg}$ DT, B6-Gsl5 TRECK-Tg mice at first showed polyuria (data not shown) and later excreted turbid urine, including a large amount of cell debris (Fig. 2A), whereas WT mice did not show these abnormal symptoms. The debris may be derived from the S3 segment because most of the debris was positive for AQP7 (Fig. 2B), which is restricted in S3 segment cells in the kidney (Ishibashi et al. 2000).

Figure 3 shows the time course of excreted urinary protein concentration following DT administration. The volume of urine changed daily, so the protein
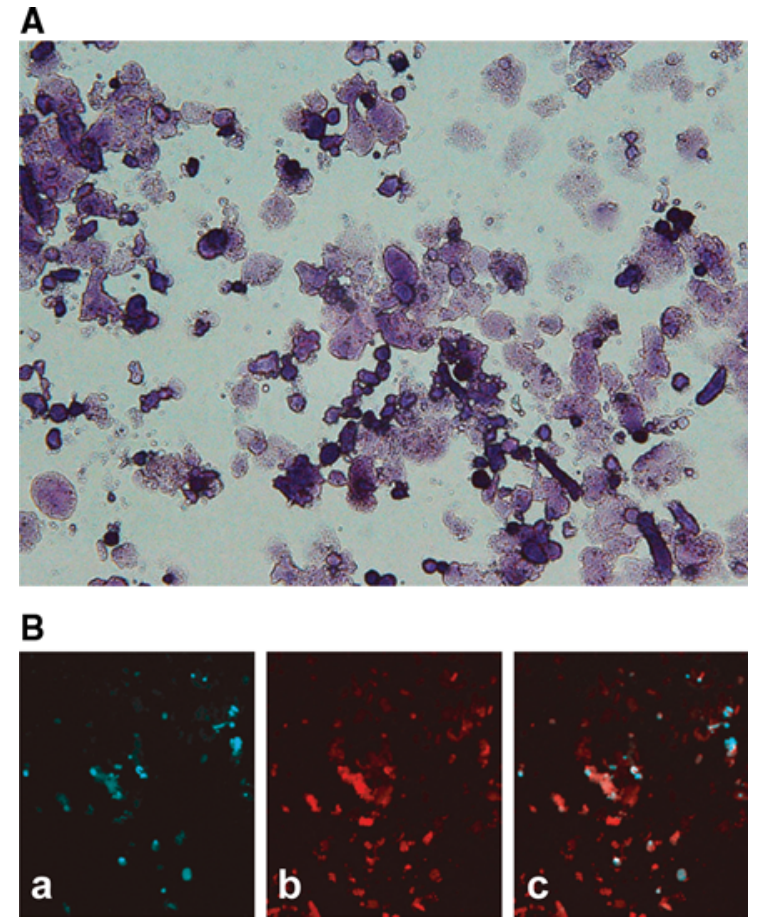

Fig. 2 Urinary sediments excreted 2 days after $50 \mu \mathrm{g} / \mathrm{kg}$ DT administration. A A urine sample was spread onto a glass slide, fixed with methanol and stained with Giemsa solution. B Another urine sample on the slide was fixed with paraformaldehyde and incubated with rabbit anti-AQP7 antibody followed by an AlexaFluor594-conjugated anti-rabbit IgG antibody (red in $b$ and $c$ ). DAPI was used to detect nuclei (blue in $a$ and $c$ ). We can find cell debris expressing AQP7 along with the intact cells in urine

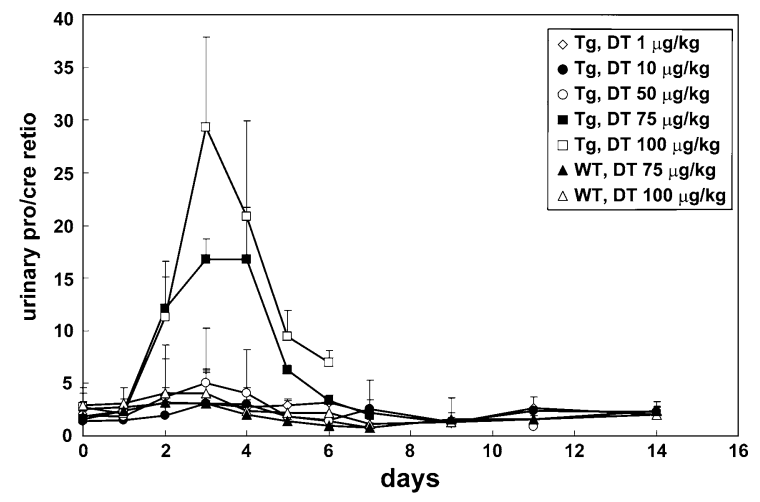

Fig. 3 Urinary excretion of proteins after DT administration. DT was injected at doses of $1(n=5), 10(n=4), 50(n=5)$, $75(n=6)$ and $100(n=5) \mu \mathrm{g} / \mathrm{kg}$ body weight for $\mathrm{Tg}$ mice and $100(n=5) \mu \mathrm{g} / \mathrm{kg}$ for WT littermates. Values were normalized with the concentration of urinary creatinine. Vertical bars are SDs 


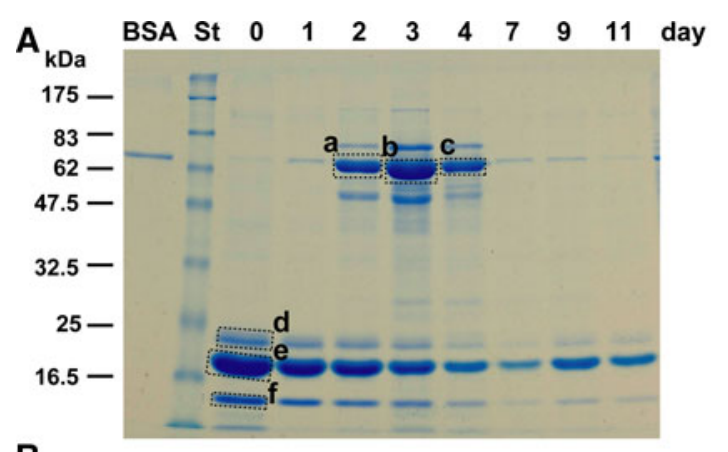

B

1 MKWVTFLLLL FVSGSAFSRG VFRREAHKSE IAHRYNDLGE QHFKGLVLIA 51 FSQYLQKCSY DEKAKLVQEV TDFAKTCYAD ESAANCDKSL HTLFGDKLCA 101 IPNLRENYGE LADCCTKOEP ERNECFLOHK DDNPSLPPFE RPEAEAMCTS

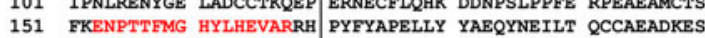
201 CLTPKLDGVK EKALVSSVRQ RMKCSSMQKF GERAFKAWAV ARLSQTFPNA 251 DFAEITKLAT DLTKVNKECC HGDLLECADD RAELAKYMCE NQATISSKLQ 301 TCCDKPLLKK AHCLSEVEHD TMPADLPAIA ADFVEDQEVC KNYAEAKDVE 351 LCTELYEYSR RHPDYSVSLI LRLAKKYEAT LEKCCAEANP PACYGTVIAE 401 FQPLVEEPKN LVKTNCDLYE KLGEYGFQNA ILVRYTQKAP QVSTPTLVEA 451 ARNLGRVGTK CCTLPEDQRL PCVEDYLSAI LNRVCLLHEK TPVSEHVTKC 501 CSGSLVERRP CFSALTVDET YVPKEFKAET FTFHSDICTL PEKEKQIKKQ 551 TALAELVKHK HKATAEQLKT VMDDFAQFLD TCCKAADKDT CFSTEGPNLV 601 TRCKDALA

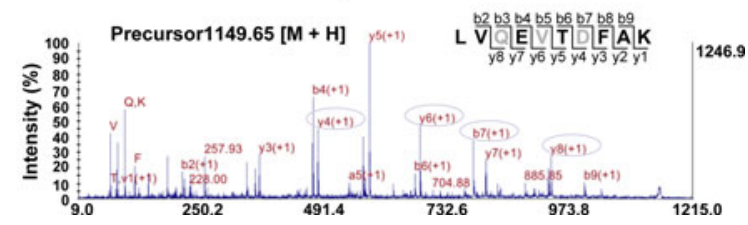

Fig. 4 Identification of mouse serum albumin excreted into the urine of a Tg mouse after DT administration. A SDSPAGE analysis of urine samples of a Tg mouse before (0) and $1-11$ days after DT administration of $50 \mu \mathrm{g} / \mathrm{kg}$. The CBBstained protein bands ( $a$ to $f$ ) were excised. The proteins in gel pieces were digested with trypsin, and the digested peptides were extracted and analyzed by a tandem mass spectrometry. $a$, $b$ and $c$ were identified as mouse serum albumin; $d$ a mouse major urinary protein, MUP3; $e$ another major urinary protein, MUP1; and $f$ a mouse $\alpha_{2 U}$ globulin V. St, Protein standards; BSA, bovine serum albumin. B Tandem mass spectrometric identification of the $65 \mathrm{kDa}$ protein (band $b$ ). Upper, the amino acid sequence of mouse serum albumin is shown. Red characters indicate the amino acid sequences determined by tandem mass spectrometric analysis. Lower, the product ion spectrum of the precursor fragment with 1149.65 of mass value obtained from band $b$. This spectrum identified the amino acid sequence LVQEVTDFAX, corresponding to amino acids 66-75 in mouse serum albumin. Amino acids Q, V and D, indicated by the product ion fragments, b7, y4, y6 and y8, are characteristic for mouse serum albumin but not for bovine or human albumin

concentrations were normalized based on urinary creatinine concentration. At high doses of DT (75 and $100 \mu \mathrm{g} / \mathrm{kg}$ ), Tg mice began excreting a large amount of protein on day 2 following DT administration. The protein concentration reached its highest level on days 3-4 and then decreased. The urinary protein/ creatinine ratio returned to normal levels 1 week after the administration of DT. Mice excrete the so-called major urinary proteins (MUPs) into their urine under normal conditions (Cavaggioni and Mucignat-Caretta 2000). Other protein bands with higher molecular weights, however, were detected on SDS-PAGE gels after DT administration (Fig. 4A). On days 2-4, a protein band of approximately $65 \mathrm{kDa}$ was detected in $\mathrm{Tg}$ mouse urine, along with the MUPs. We analyzed the protein by tandem mass spectrometry after digesting with trypsin and determined that the protein was serum albumin (Fig. 4B). The excretion of serum albumin into urine suggests that the S3 segment plays an important role in its reabsorption.

$\mathrm{Tg}$ mice administered these high doses of DT eventually developed oliguria. Four of five mice that received $100 \mu \mathrm{g} / \mathrm{kg}$ died on day 6 , and two of six mice that received $75 \mu \mathrm{g} / \mathrm{kg}$ also died on day 6 . These mice lost $20 \%$ of their body weight after DT administration. The oliguria was considered the result of intratubular obstructive nephropathy, followed by severe damage to the $\mathrm{S} 3$ segment (see the section on histological observations and Fig. 5). The surviving mice also suffered proteinuria; however, they recovered from the abnormal symptoms and lived for more than 2 months, although lesions in the S3 segment were still evident 2 months later.

When administered DT at a dose of $50 \mu \mathrm{g} / \mathrm{kg}$, Tg mice showed a wide range of individual differences in the excretion of serum albumin (Fig. 3), while the amount of MUPs and the proportion among MUPs did not differ during the 2 week period after DT administration (data not shown). On the other hand,

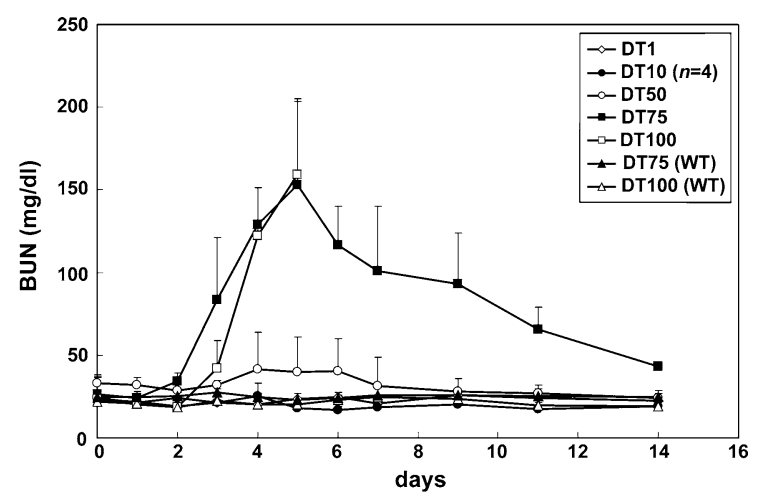

Fig. 5 Concentration of blood urea nitrogen (BUN) after DT administration Vertical bars are SDs 
the average concentration of BUN was elevated but recovered to normal levels 1 week after DT administration (Fig. 5). Lower doses of DT (1 and $10 \mu \mathrm{g} / \mathrm{kg}$ ) did not cause any substantial effects in the Tg mice, which showed BUN levels similar to those of WT mice. When $\mathrm{Tg}$ mice were injected with 75 and $100 \mu \mathrm{g} / \mathrm{kg}$ DT, their BUN levels began to increase on day 3 after the administration of DT and peaked on day 5 at a level of $150 \mathrm{mg} / \mathrm{dl}$. Although the four surviving Tg mice at a dose of $75 \mu \mathrm{g} / \mathrm{kg}$ (Fig. 5) and the one surviving $\mathrm{Tg}$ mouse at a dose of $100 \mu \mathrm{g} / \mathrm{kg}$ DT (data not shown) still showed higher BUN concentrations than WT mice for 2 weeks, they recovered from the injury, and their BUN values were within the normal range on day 28 (data not shown).

Histological analysis exhibited severe injury in the kidneys of the Tg mice, especially in the S3 segment, at a dose of $50 \mu \mathrm{g} / \mathrm{kg}$. On day 3 , the proximal tubule cells of the $\mathrm{Tg}$ mice were significantly disorganized (Fig. 6A-a), whereas the proximal tubule cells of the WT mice did not show any abnormalities (Fig. 6A-b). Proximal tubule epithelial cells with thick cytoplasm and a well-developed microvillous membrane seemed intact in the kidneys of WT mice. The Tg mice that died on day 6 after a dose of $100 \mu \mathrm{g} / \mathrm{kg}$ DT appeared to be more intensely damaged than those at a dose of $50 \mu \mathrm{g} / \mathrm{kg}$ (Fig. 6B). A lot of casts were observed (Fig. 6B-a, arrows). In proximal tubules, the S3 segment and the S1 and S2 segments were injured, although the most severe acute tubular injury was observed in S3 segment cells (Fig. 6B-b, arrowheads). In the S3 segment, tubular cells were exfoliated into the tubular lumina, and some tubular basement membranes were denuded (Fig. 6B-c, white arrow). Cast formation was detected in distal tubule cells (Fig. 6B-c, black arrow). On the other hand, glomeruli did not show any damage (Fig. 6B-d, arrow). At a dose of $75 \mu \mathrm{g} / \mathrm{kg}$, we observed regenerating features in proximal tubules of $\mathrm{Tg}$ mice on day 14 (Fig. 6C). While the distal tubules had no casts and the brush border of the S1 and S2 segments was recovered, we observed both damaged S3 segments (Fig. 6C-a, black arrowheads) and regenerated S3 tubules (Fig. 6C-b, white arrowheads).

Similar results were observed by immunohistochemistry (Fig. 7). AQP7, which is localized exclusively to the S3 segment in the kidney, almost disappeared from $\mathrm{Tg}$ mice treated with $50 \mu \mathrm{g} / \mathrm{kg}$ DT

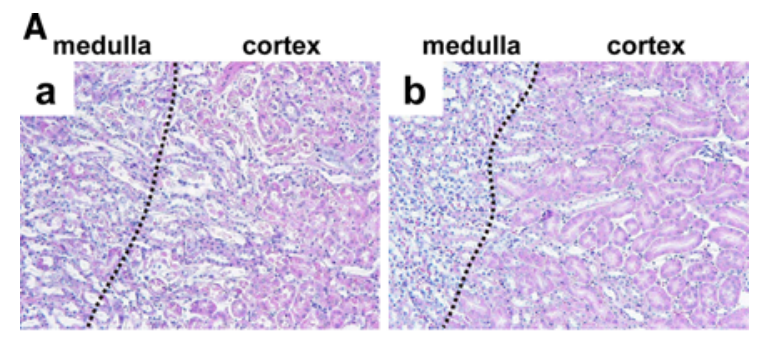

B

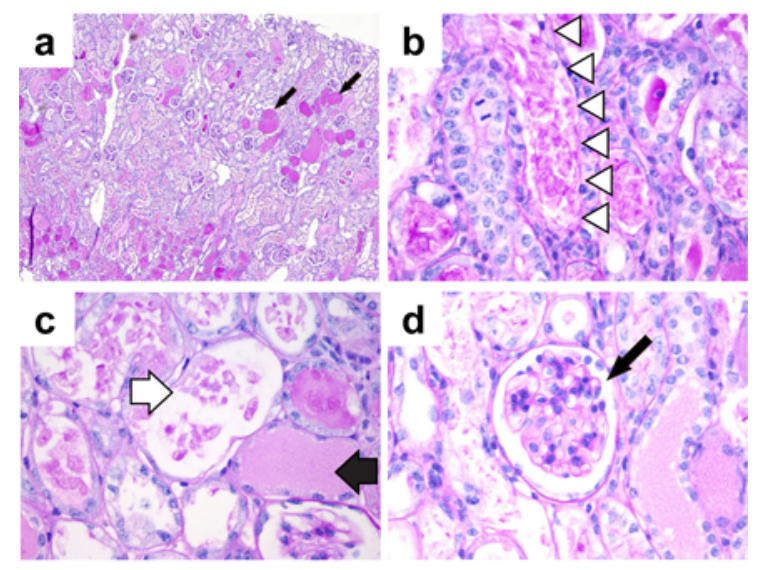

C

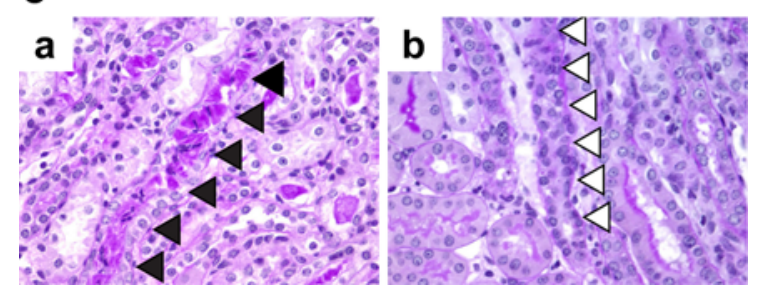

Fig. 6 Histochemical studies after DT administration. A The boundary region between the cortex and medulla on day 3 after $50 \mu \mathrm{g} / \mathrm{kg}$ DT administration in $\mathrm{Tg}(a)$ and WT $(b)$ mice. Dotted line shows the border between the cortex and the medulla. Stained with HE. B PAS stained sections of the kidney of a Tg mouse died on day 6 after $100 \mu \mathrm{g} / \mathrm{kg}$ DT administration. (a) In proximal tubules, not only the $\mathrm{S} 3$ segment but also the more proximal segments $\mathrm{S} 1$ and $\mathrm{S} 2$ were injured. Cast formation (arrows) was observed in the distal tubules. (b) White arrowheads indicate acute tubular injury observed in the S3 segment. (c) In the S3 segment, tubular cells were exfoliated into lumen, and some tubules were denuded (white arrow). Some distal tubules were stuck with casts (black arrow). (d) The glomerulus (arrow) seemed to be intact. C PAS stained sections of Tg mouse kidney on day 14 after $75 \mu \mathrm{g} / \mathrm{kg}$ DT administration. Both damaged $(a)$ and regenerated (b) S3 segments were observed (arrowheads)

(Fig. 6) on day 3, confirming that their S3 segment cells were heavily destroyed. Megalin, which is expressed in proximal tubules including the S3 segment, was partially disappeared or decreased. 


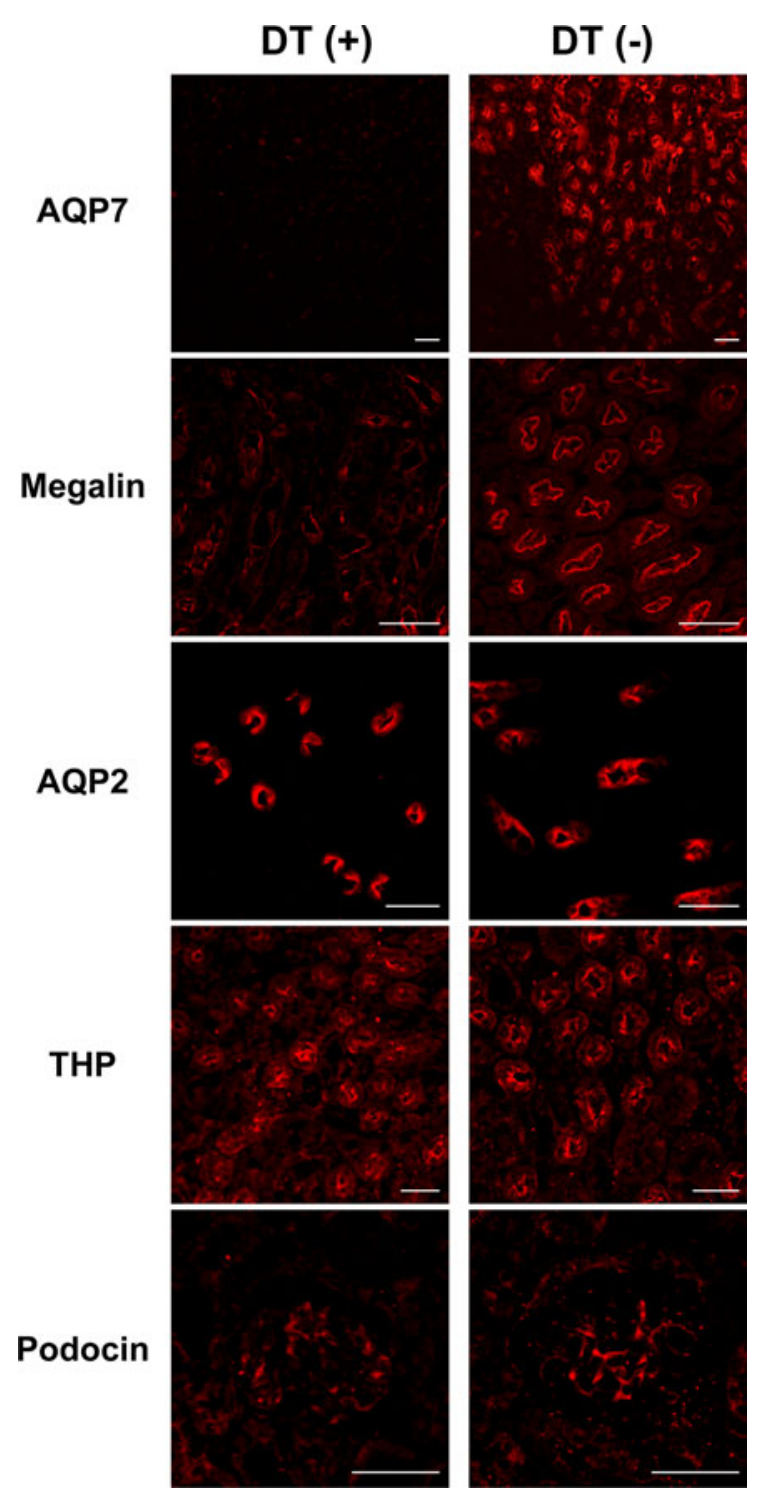

Fig. 7 Immunohistochemistry of the kidney after DT administration. AQP7 localized in the S3 segment cells vanished from the kidney of a Tg mouse on day 3 after $75 \mu \mathrm{g} / \mathrm{kg}$ of DT administration (DT $(+)$ ), whereas a control mouse without DT administration (DT $(-)$ ) showed no abnormality. In the $\mathrm{Tg}$ mouse, megalin expressed in proximal tubule cells also partially disappeared. AQP2 in the collecting duct cells, THP in TAL cells, podocin in a glomerulus were not affected. Scale bars $=50 \mu \mathrm{m}$

On the other hand, marker proteins of other segments, such as THP for thick ascending limb (TAL) cells, AQP2, located in collecting ducts, and podocin for glomerulus did not diminish, and these cells were not affected by DT administration. In the control mice
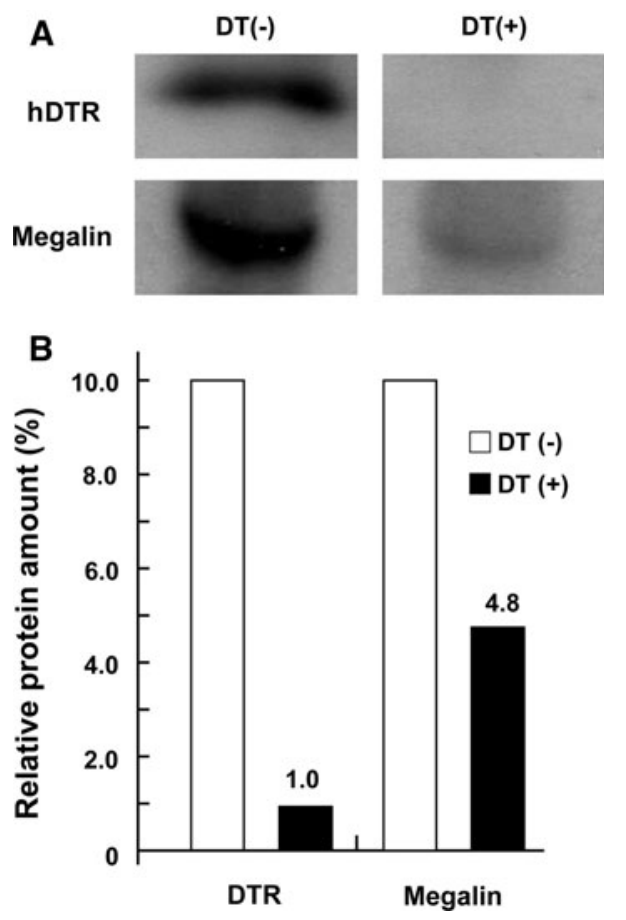

Fig. 8 Western blotting analysis of the kidney after DT administration. A Western blot analysis of the membrane fractions prepared from kidney of DT $(+)$ and DT (-) mouse using anti-hDTR (top) and anti-megalin (bottom) antibodies. For hDTR, the equivalent amount of one-eighth of the kidney was applied on the gel. For megalin, $5 \mu \mathrm{g}$ of the kidney membrane protein was applied. B Densitometric quantification of hDTR and megalin expression levels in A. DT (+), a Tg mouse on day 3 after $75 \mu \mathrm{g} / \mathrm{kg}$ of DT administration; DT (-), a control mouse without DT administration

without DT administration, neither S3 segment nor other segment cells were damaged. To quantitate the effect of DT on megalin and hDTR, we analyzed these proteins by western blotting. Figure 8 showed that megalin was decreased about $50 \%$ of the control, however, was less affected than hDTR. Megalin expressed in S1 and S2 segment seemed to be remained.

These results suggest that DT administration effectively eliminated kidney S3 segment cells in the $\mathrm{Tg}$ mice in an S3 segment cell-specific manner and that the Tg mice developed symptoms of AKI.

\section{Discussion}

S3 segment cells are susceptible to ischemia but also have a remarkable ability to restore their structure 
and function (Sharples 2007). To induce damage in the S3 segment cells, experimental surgery on renal arteries or injection of nephrotoxic substances such as cisplatin or uranyl acetate have been employed (Leibbrandt et al. 1995; Sun et al. 2000; Park et al. 2001; Kobayashi et al. 2008; Wainford et al. 2008). The effects of ischemia or chemical treatments, however, are not specific to the S3 segment, as other parts of the kidney and other organs can also be affected by these treatments. The precise mechanisms and processes of regeneration after ischemic acute renal injury have been a matter of controversial discussions (Kitamura et al. 2005; Duffield and Bonventre 2005; Humphreys and Bonventre 2008; Gupta and Rosenberg 2008). To clarify the factors involved in S3 segment-specific lesions and their repair, a more $\mathrm{S} 3$ segment-restricted model system is necessary. Our Tg mouse described in this paper is a novel model to elucidate the important factors functioning in the processes of injury and recovery of S3 segment cells in AKI.

The established B6-Gsl5 TRECK-Tg mouse expresses hDTR, which acts as a receptor for DT, exclusively in S3 segment cells. By administering DT, we ablated S3 segment cells specifically and dose dependently. Biochemical analyses detected several abnormalities in the urine and serum samples of the treated Tg mice, e.g., high BUN values (Fig. 5), high urinary protein/creatinine ratio (Fig. 3), and transient excretion of serum albumin (Fig. 4). These data indicate that the $\mathrm{Tg}$ mice suffered impaired renal function and, in particular, deficiency of the proximal tubule cells. Because proximal tubules reabsorb serum albumin, which passes through the glomerular capillary wall under ordinary conditions (Lazzara and Deen 2007; Russo et al. 2007), the disrupted S3 segment cells could not completely reabsorb albumin. Consequently, it was excreted into the urine, even though the glomeruli were intact.

Using the podocin promoter, one report has applied the TRECK system on a kidney disease model (Wharram et al. 2005). The generated transgenic rats specifically expressed hDTR on podocytes and administration of DT into these rats resulted in dose-dependent podocyte depletion from glomeruli and led to glomerulosclerosis. These rats showed transient proteinuria as well as our Tg mice; however, they expressed much higher values of protein/creatinine ratio and more prolonged excretion than our $\mathrm{Tg}$ mice, suggesting that the glomeruli of our $\mathrm{Tg}$ mice were not affected. Histopathological (Fig. 6) and immunohistochemical (Fig. 7) analyses of our $\mathrm{Tg}$ mouse kidneys after DT administration confirmed that the proximal tubular cells on the boundary between the cortex and medulla were severely damaged and that the S3 segment-specific AQP7 was disappeared from this region. These results clearly demonstrate that our Tg mouse is an excellent model for AKI due to S3 segment-specific damage and is useful for elucidating the pathophysiological functions of the epithelial cells in the S3 segment.

The TRECK system is an effective method to analyze the function of cells in vivo. By administering DT to the transgenic mice, we can induce the depletion of target cells expressing hDTR under the control of a cell-specific promoter/enhancer at any desired age of the $\mathrm{Tg}$ mouse to identify the in vivo function of the cells. To date, several cell-specific promoters have been used to generate $\mathrm{Tg}$ mice for TRECK experiments, such as for vascular smooth muscle cells (Clarke et al. 2006), macrophages (Miyake et al. 2007), and Foxp3 regulatory T cells (Lahl et al. 2007). These mice showed cell-specific ablation and injury after DT administration, demonstrating that the TRECK-Tg mice are promising models for evaluating in vivo functions of one specific cell or a cell lineage of interest.

We have identified Gsl5 as a cis-regulatory element for $\mathrm{c} 2 \mathrm{GnT}$ in the mouse kidney on the basis of polymorphic expression of kidney glycolipids among inbred strains of mice (Sekine et al. 2001, 2002, 2006). Gsl5 is indispensable for the transcription of kidney-specific mRNA for the $\mathrm{c} 2 \mathrm{GnT}$ gene and, hence, for the expression of a unique glycolipid. Neither this glycolipid nor the kidney-type mRNA of c2GnT has been found thus far in tissues other than kidney. Using Gsl5, we expressed EGFP protein in S3 segment cells of Gsl5-EGFP transgenic mice (Sekine et al. 2006) Although Gsl5 does not have particularly strong promoter/enhancer activity to express hDTR in the S3 segment, as judged by IP/ WB and immunohistochemical analyses (Fig. 1C, D), it was sufficient to provide susceptibility to DT in the S3 segment, given that a single incorporated molecule of DT is enough to kill a cell (Yamaizumi et al. 1978). AQP7, which is a member of the aquaporin family and is localized to the S3 segment of the kidney (Ishibashi et al. 2000), may have a much 
stronger promoter/enhancer element; however, AQP7 is not restricted to the kidney and is also expressed in testis and adipose tissues (Hara-Chikuma et al. 2005). For specificity to the $\mathrm{S} 3$ segment, Gsl5 is preferable.

Using our B6-Gsl5 TRECK Tg mice, we obtained two lines of valuable evidence to prove the importance of the S3 segment. First, depletion of the S3 segment itself induced symptoms of AKI that are found in clinical cases. In experimental ischemic AKI, damage has been reported mainly in the S3 segment, simply because the S3 segment is vulnerable to ischemia. We confirmed that disruption of the S3 segment induced AKI and that S1, S2 and the distal segments likely showed only secondary damage, while the S3 segment showed severe injury. Another important finding was obtained by comparing our results with those presented in a paper describing the deletion of TAL cells (Srichai et al. 2008). Tg mice expressing the herpes simplex virus 1 thymidine kinase gene under the control of the TALspecific THP promoter showed apoptosis only in TAL cells after administration of ganciclovir and caused elevation of BUN values and oliguria. It is interesting that the mice showed impairment in urinary concentrating ability. On the other hand, selective depletion of the S3 segment in our model caused excretion of serum albumin into the urine. Although both mice are models for AKI, they each expressed different segment-specific features.

In conclusion, we generated a novel $\mathrm{Tg}$ mouse line expressing hDTR exclusively in the S3 segment of the kidney. We confirmed that the Tg mice suffer AKI originating from S3 segment-specific depletion following DT administration. Furthermore, we could regulate the extent of injury in a dose-dependent manner. Because of its strict tissue specificity, this $\mathrm{Tg}$ mouse offers an excellent model to elucidate the pathophysiological functions of epithelial cells in the S3 segment and investigate the factors responsible for AKI and rapid recovery.

Acknowledgments We are grateful to Satomi Yamada and Sumiyo Takahama for their technical assistance. This work was supported by the Promotion of Basic Research Activities for Innovative Biosciences (PROBRAIN) (H.Y.) and partly by a grant-in-aid (A) from the Japanese Society for the Promotion of Science (H.Y. and K.K.), a Takeda Science Foundation research grant (H.Y.), and a research grant from the ministry of Health, Labour and Welfare in Japan (M.H.).
Conflict of interest The authors declare that they have no conflict of interest interests.

Open Access This article is distributed under the terms of the Creative Commons Attribution Noncommercial License which permits any noncommercial use, distribution, and reproduction in any medium, provided the original author(s) and source are credited.

\section{References}

Abraham JA, Damm D, Bajardi A, Miller J, Klagsbrun M, Ezekowitz RA (1993) Heparin-binding EGF-like growth factor: characterization of rat and mouse cDNA clones, protein domain conservation across species, and transcript expression in tissues. Biochem Biophys Res Commun 190(1):125-133

Akita Y, Kawasaki H, Imajoh-Ohmi S, Fukuda H, Ohno S, Hirano H, Ono Y, Yonekawa H (2007) Protein kinase C $\varepsilon$ phosphorylates keratin 8 at Ser8 and Ser23 in $\mathrm{GH}_{4} \mathrm{C}_{1}$ cells stimulated by thyrotropin-releasing hormone. FEBS J 274:3270-3285

Cavaggioni A, Mucignat-Caretta C (2000) Major urinary proteins, $\alpha_{2 \mathrm{U}}$-globulins and aphrodisin. Biochim Biophys Acta 1482:218-228

Clarke MCH, Figg N, Maguire JJ, Davenport AP, Goddard M, Littlewood TD, Bennett MAR (2006) Apoptosis of vascular smooth muscle cells induces features of plaque vulnerability in atherosclerosis. Nat Med 12:1075-1080

Cristofori P, Zanetti E, Fregona D, Piaia A, Trevisan A (2007) Renal proximal tubule segment-specific nephrotoxicity: an overview on biomarkers and histopathology. Toxicol Pathol 35:270-275

Duffield JS, Bonventre JV (2005) Kidney tubular epithelium is restored without replacement with bone marrow-derived cells during repair after ischemic injury. Kidney Int 68:1956-1961

Gupta S, Rosenberg ME (2008) Do stem cells exist in the adult kidney? Am J Nephrol 28:607-613

Hara-Chikuma M, Sohara E, Rai T, Ikawa M, Okabe M, Sasaki S, Uchida S, Verkman AS (2005) Progressive adipocyte hypertrophy in aquaporin-7-deficient mice. J Biol Chem 280:15493-15496

Higashiyama S, Lau K, Besner GE, Abraham JA, Klagsbrun M (1992) Structure of heparin-binding EGF-like growth factor. J Biol Chem 267:6205-6212

Honjo T, Nishizuka Y, Hayaishi O, Kato I (1968) Diphtheria toxin-dependent adenosine diphosphate ribosylation of aminoacyl transferase II and inhibition of protein synthesis. J Biol Chem 243:3553-3555

Hooper KP, Eidels L (1996) Glutamic acid 141 of the diphtheria toxin receptor (HB-EGF precursor) is critical for toxin binding and toxin sensitivity. Biochem Biophysc Res Commun 220:675-680

Humphreys BD, Bonventre JV (2008) Mesenchymal stem cells in acute kidney injury. Annu Rev Med 59:311-325 
Ishibashi K, Imai M, Sasaki S (2000) Cellular localization of aquaporin 7 in the rat kidney. Exp Nephrol 8:252-257

Kitamura S, Yamasaki Y, Kinomura M, Sugaya T, Sugiyama H, Maeshima Y, Makino H (2005) Establishment and characterization of renal progenitor like cells from S3 segment of nephron in rat adult kidney. FASEB J 19:1789-1797

Kobayashi T, Terada Y, Kuwana H, Tanaka H, Okado T, Kuwahara M, Tohda S, Sakano S, Sasaki S (2008) Expression and function of the Delta-1/Notch-2/Hes-1 pathway during experimental acute kidney injury. Kidney Int 73:1240-1250

Lahl K, Loddenkemper C, Drouin C, Freyer J, Arnason J, Eberl G, Hamann A, Wagner H, Huehn J, Sparwasser T (2007) Selective depletion of Foxp3+ regulatory $\mathrm{T}$ cells induces a scurfy-like disease. J Exp Med 204:57-63

Lazzara MJ, Deen WM (2007) Model of albumin reabsorption in the proximal tubule. Am J Physiol Renal Physiol 292:F430-F439

Leibbrandt MEI, Wolfgang GHI, Metz AL, Ozobia AA, Haskins JR (1995) Critical subcellular targets of cisplatin and related platinum analogs in rat renal proximal tubule cells. Kidney Int 48:761-770

Maeshima A, Yamashita S, Nojima Y (2003) Identification of renal progenitor-like tubular cells that participate in the regeneration processes of the kidney. J Am Soc Nephrol 14:3138-3146

Mitamura T, Higashiyama S, Taniguchi N, Klagsbrun M, Mekada E (1995) Diphtheria toxin binds to the epidermal growth factor (EGF)-like domain of human heparin-binding EGFlike growth factor/diphtheria toxin receptor and inhibits specifically its mitogenic activity. J Biol Chem 270:1015-1019

Miyake Y, Kaise H, Isono K, Koseki H, Kohno K, Tanaka M (2007) Protective role of macrophages in noninflammatory lung injury caused by selective ablation of alveolar epithelial type II cells. J Immunol 178:5001-5009

Park KM, Chen A, Bonventre JV (2001) Prevention of kidney ischemia/reperfusion -induced functional injury and JNK, p38, and MAPK kinase activation by remote ischemic pretreatment. J Biol Chem 276:11870-11876

Russo LM, Sandoval RM, McKee M, Osicka TM, Collins AB, Brown D, Molitoris BA, Comper WD (2007) The normal kidney filters nephrotic levels of albumin retrieved by proximal tubule cells: retrieval is disrupted in nephrotic states. Kidney Int 71:504-513

Saito M, Iwawaki T, Taya C, Yonekawa H, Noda M, Inui Y, Mekada E, Kimata Y, Tsuru A, Kohno K (2001)
Diphtheria toxin receptor-mediated conditional and targeted cell ablation in transgenic mice. Nature Biotechnol 19:746-750

Sekine M, Taya C, Kikkawa Y, Yonekawa H, Takenaka M, Matsuoka Y, Imai E, Izawa M, Kannagi R, Suzuki A (2001) Regulation of mouse kidney tubular epithelial cellspecific expression of core 2 GlcNAc transferase. Eur J Biochem 268:1129-1135

Sekine M, Kikkawa Y, Takahama S, Tsuda K, Yonekawa H, Suzuki A (2002) Phylogenetic development of a regulatory gene for the core 2 GlcNAc transferase in Mus musculus. J Biochem 132:387-393

Sekine M, Taya C, Shitara H, Kikkawa Y, Akamatsu N, Kotani M, Miyazaki M, Suzuki A, Yonekawa H (2006) The cisregulatory element Gs15 is indispensable for proximal straight tubule cell-specific transcription of core $2 \beta-1$, $6-\mathrm{N}$-acetylglucosaminyltransferase in the mouse kidney. J Biol Chem 281:1008-1015

Sharples EJ (2007) Acute kidney injury: stimulation of repair. Curr Opin Crit Care 13:652-655

Srichai MB, Hao C, Davis L, Golovin A, Zhao M, Moeckel G, Dunn S, Bulus N, Harris RC, Zent R, Breyer MD (2008) Apoptosis of the thick ascending limb results in acute kidney injury. J Am Soc Nephrol 19:1538-1546

Sun DF, Fujigaki Y, Fujimoto T, Yonemura K, Hishida A (2000) Possible involvement of myofibroblasts in cellular recovery of uranyl acetate-induced acute renal failure in rats. Am J Pathol 157:1321-1335

Wainford RD, Weaver RJ, Stewart KN, Brown P, Hawksworth GM (2008) Cisplatin nephrotoxicity is mediated by gamma glutamyltranspeptidase, not via a C-S lyase governed biotransformation pathway. Toxicology 249:184-193

Wharram BL, Goyal M, Wiggins JE, Sanden SK, Hussain S, Filipiak WE, Saunders TL, Dysko RC, Kohno K, Holzman LB, Wiggins RC (2005) Podocyte depletion causes glomerulosclerosis: Diphtheria toxin-induced podocyte depletion in rats expressing human diphtheria toxin receptor transgene. J Am Soc Nephrol 16:2941-2952

Witzgall R, Brown D, Schwarz C, Bonventre JV (1994) Localization of proliferating cell nuclear antigen, vimentin, c-Fos and clusterin in the postischemic kidney. J Clin Invest 93:2175-2188

Yamaizumi M, Mekada E, Uchida T, Okada Y (1978) One molecule of diphtheria toxin fragment A introduced into a cell can kill the cell. Cell 15:245-250 